\title{
Non-activated APJ suppresses the angiotensin II type 1 receptor, whereas apelin-activated APJ acts conversely
}

\author{
Xiao Sun ${ }^{1}$, Shinichiro Iida ${ }^{2}$, Ayumu Yoshikawa ${ }^{3}$, Rina Senbonmatsu ${ }^{4}$, Kazuhito Imanaka ${ }^{1}$, Kei Maruyama ${ }^{3}$, \\ Shigeyuki Nishimura ${ }^{2}$, Tadashi Inagami ${ }^{5}$ and Takaaki Senbonmatsu ${ }^{3}$
}

Apelin and its G-protein-coupled receptor APJ are potent regulators of the cardiovascular system. Recent studies have suggested that apelin-APJ reverses the function of angiotensin II (Ang II)-the Ang II type 1 receptor $\left(\mathrm{AT}_{1}\right)$. However, the mechanism remains unclear because of the accumulating evidences that apelin-APJ may contribute to both cardioprotection and pathological progression. In human embryonic kidney 293 cells, we found that coexpression with APJ significantly suppressed the phosphorylation of extracellular signal-regulated kinases 1/2 (ERK1/2) induced by Ang II-AT 1 , whereas apelin abolished this attenuation through activated APJ independently of its heterodimerization. Pretreatment with the Gi/o-specific inhibitor pertussis toxin (PTX) restituted the ERK1/2 phosphorylation level similar to that found with $\mathrm{AT}_{1}$ and APJ coexpression without apelin stimulation. In contrast, coexpression of the beta-2-adrenergic receptor or the pharmacologically non-activated Ang II type 2 receptor $\left(\mathrm{AT}_{2}\right)$ pretreated with the $\mathrm{AT}_{2}$-specific antagonist, $\mathrm{PD} 123319$, did not affect ERK1/2 phosphorylation through $\mathrm{AT}_{1}$. Pretreatment with $30 \mathrm{~nm}$ of the $\mathrm{AT}_{1}$ blocker (ARB) TA-606A suppressed $50 \%$ of the $\mathrm{AT}_{1}$-mediated ERK1/2 phosphorylation, whereas $30 \mathrm{nM}$ of TA-606A achieved 75\% suppression when the non-activated APJ was coexpressed without ligand or PTX. However, $120 \mathrm{~nm}$ of TA-606A failed to reach the target phosphorylation when it was coexpressed with activated APJ with apelin. Based on these results, we demonstrated that non-activated APJ may suppress Ang II-AT 1 signaling, whereas this ligand-independent function was diminished with apelin activation. These results may be relevant to the potential contribution of apelin-APJ to ARB treatment in the clinical realm.

Hypertension Research (2011) 34, 701-706; doi:10.1038/hr.2011.19; published online 17 March 2011

Keywords: apelin; APJ; angiotensin II type 1 receptor; angiotensin II type 1 receptor blocker

\section{INTRODUCTION}

The 380-amino acid, 7-transmembrane domain G-protein-coupled receptor (GPCR) called APJ, which is most closely related to the angiotensin II (Ang II) type 1 receptor $\left(\mathrm{AT}_{1}\right)$ but does not bind to Ang II, was identified through the Human Genome Project. ${ }^{1}$ In 1998, its endogenous ligand called apelin was identified. ${ }^{2}$ Apelin and APJ are highly expressed in cardiomyocytes, endothelial cells and vascular smooth muscle cells (VSMC). Apelin activates APJ, leading to intracellular signaling both locally and in an endocrine fashion. ${ }^{3,4}$ Since its discovery, the apelin-APJ system has emerged as an important regulator of cardiovascular homeostasis and may have a critical role in the pathophysiological development of cardiovascular diseases; it has also been regarded as a new therapeutic research and drug design target. ${ }^{5-11}$ The functions of apelin-APJ appear to be complex and have yet to be defined by consensus. The accumulating evidences have indicated that the functions of apelin-APJ may be dependent on its target organs and its physiological/pathophysiological state. The experiments performed using cardiomyocytes have shown that
apelin-APJ evokes contractility and promotes growth; however, in the vasculature, apelin-APJ acts as a vasodilator as part of its cardioprotective function under physiological but not pathophysiological conditions. $^{5,8}$

Recent studies have revealed that APJ and $\mathrm{AT}_{1}$ have similar expression patterns in a number of physiological and pathophysiological states, and apelin-APJ contributes to opposing effects against Ang $\mathrm{II}-\mathrm{AT}_{1} \cdot{ }^{10,11}$ Blood pressure in $\mathrm{AT}_{1}$ and APJ double-knockout mice was significantly elevated compared with that in $\mathrm{AT}_{1}$ knockout mice. ${ }^{11}$ Chun et al. reported that apelin-APJ antagonizes Ang II-AT ${ }_{1}$ by increasing nitric oxide $(\mathrm{NO})$ production via intracellular signaling in mouse models of atherosclerosis. ${ }^{12}$ However, researchers have not yet concluded that the apelin-APJ system is a counterpart of the Ang II-AT ${ }_{1}$ system. In contrast to Chun et al., Hashimoto et al. demonstrated that in mouse models of atherosclerosis, apelin-APJ aggravates oxidative stress through increasing the nicotinamide adenine dinucleotide phosphate oxidase subunits, which are major sources of superoxide anion in the cardiovascular system. ${ }^{13}$ Similarly,

${ }^{1}$ Department of Cardiovascular Surgery, Saitama Medical Center, Saitama, Japan; ${ }^{2}$ Division of Cardiovascular Medicine, Department of Cardiovascular Medicine, Saitama International Medical Center, Saitama, Japan; ${ }^{3}$ Department of Pharmacology, Saitama Medical University, Saitama, Japan; ${ }^{4}$ Department of Neuroscience, Bates College, Lewiston, ME, USA and ${ }^{5}$ Department of Biochemistry, Vanderbilt University School of Medicine, Nashville, TN, USA

Correspondence: Dr T Senbonmatsu, Department of Pharmacology, Saitama Medical University, 38 Morohongo Moroyama-machi, Iruma-gun, Saitama 350-0495, Japan. E-mail: senbont@saitama-med.ac.jp

Received 2 September 2010; revised 17 December 2010; accepted 26 December 2010; published online 17 March 2011 
Liu et al. reported that apelin promotes VSMC proliferation through the phosphoinositide 3-kinase/Akt signaling pathway. ${ }^{14}$ These results indicate that apelin-APJ may have growth-promoting effects similar to those of Ang II-AT ${ }_{1}$ in the pathological development of cardiovascular diseases. Taken together, all of these results convincingly demonstrate that apelin-APJ may have dual functions. However, the specific correlation between both GPCRs' functions still remains controversial. In the present study, we investigated the relationship among apelin, APJ, Ang II and $\mathrm{AT}_{1}$ in an in vitro experimental model. APJ alone but not apelin-APJ may be a crucial therapeutic candidate in inhibiting Ang II-AT ${ }_{1}$.

\section{METHODS}

\section{Reagents}

The following reagents and antibodies were purchased: Ang II (Sigma-Aldrich, St Louis, MO, USA); anti-phosphorylated extracellular signal-regulated kinases 1/2 (anti-pERK1/2) antibody; anti-AT ${ }_{1}$ antibody; anti-angiotensin II type 2 receptor (anti-AT ${ }_{2}$ ) antibody; anti-APJ antibody (Santa Cruz Biotechnology, Santa Cruz, CA, USA); anti-beta-2-adrenergic receptor (anti- $\beta_{2} \mathrm{AR}$ ) antibody (Abnova, Taipei, Taiwan); anti-extracellular signal-regulated kinases 1/2 (antiERK1/2) antibody; anti-pAkt antibody; anti-Akt antibody (Cell Signaling Technology, Boston, MA, USA); pertussis toxin (PTX; Seikagaku, Tokyo, Japan); and the $\mathrm{AT}_{2}$-specific antagonist, PD123319 (Sigma-Aldrich). The $\mathrm{AT}_{1}$ blocker (ARB) TA-606A was a gift from the Mitsubishi Tanabe Pharmaceutical company (Osaka, Japan). We also purchased pyroglutamyl (Glp-1) apelin-13 (American Peptide Company, Vista, CA, USA) and used this as apelin.

\section{Vector construction}

The construction of the human full-length $\mathrm{AT}_{1}, \mathrm{APJ}, \mathrm{AT}_{2}$ and $\beta_{2} \mathrm{AR}$ were performed by PCR amplification using the human Multiple Tissue cDNA panel I (Clontech Laboratories, Palo Alto, CA, USA). The construction of the doublecassette vector system was previously described. ${ }^{15-17}$ Myc tag was inserted into pcDNA5/FRT/TO (Invitrogen, Carlsbad, CA, USA) to construct pcDNA5/FRT/ TO-myc-His. Then, elongation factor $1 \alpha(E F 1 \alpha)$ promoter, multiple cloning sites and bovine growth hormone site were inserted into pcDNA5/FRT/ TO-myc-His. Finally, pcDNA5/FRT/TO-EF1 $\alpha$-V5-His-CMV-myc-His, which contained two expression cassettes, was constructed. Thus, the vectors that expressed $\mathrm{AT}_{1}$-myc-His; $\mathrm{APJ}-\mathrm{V} 5$-His; $\mathrm{AT}_{1}$-myc-His plus APJ-V5-His; $\mathrm{AT}_{1}$-mycHis plus $\mathrm{AT}_{2}$-V5-His; and $\mathrm{AT}_{1}$-myc-His plus $\beta_{2} \mathrm{AR}-\mathrm{V} 5$-His were created.

\section{Cell culture and transfection}

Human embryonic kidney (HEK) 293 cells were grown in Dulbecco's modified Eagle's medium supplemented with $10 \%$ fetal bovine serum at $37^{\circ} \mathrm{C}$. Transient transfection of HEK293 cells was performed using a Fugene6 reagent (Roche Molecular Biochemicals, Indianapolis, IN, USA) as per the manufacturer's protocols. At $30 \mathrm{~h}$ after transfection, the cells were serum starved for $6 \mathrm{~h}$ before agonist stimulation. For PTX pretreatment, the cells were incubated with $200 \mathrm{ng} \mathrm{ml}^{-1}$ of PTX for $3 \mathrm{~h}$. For PD123319 pretreatment, the cells were incubated with $10 \mu \mathrm{M}$ of PD123319 for $1 \mathrm{~h}$. The cells were pretreated with 15, 30,60 and $120 \mathrm{~nm}$ of TA-606A for $90 \mathrm{~min}$ as previously described. ${ }^{18}$

\section{Immunoblotting}

The cells were stimulated with Ang II (100 nM); apelin (100 nM) or Ang II plus apelin at $37^{\circ} \mathrm{C}$ in serum-free media after preincubation with the indicated reagents. Then, cells were lysed in an ice-cold RIPA buffer $\left(50 \mathrm{mmoll}^{-1}\right.$ Tris$\mathrm{HCl}$, pH 8.0, $150 \mathrm{mmoll}^{-1} \mathrm{NaCl}, 5 \mathrm{mmoll}^{-1}$ EDTA, $1 \%$ Nonidet P-40, $0.1 \%$ SDS and $0.5 \%$ deoxycholate) containing $100 \mathrm{mmoll}^{-1}$ phenylmethylsulfonyl fluoride and protease inhibitor cocktail (Nacalai Tesque, Kyoto, Japan) at $4{ }^{\circ} \mathrm{C}$ and centrifuged at $20000 \mathrm{~g}$ for $60 \mathrm{~min}$ at $4{ }^{\circ} \mathrm{C}$. The protein concentration of the supernatant was determined using a BCA protein assay kit (Pierce, Rockford, IL, USA). Then, $4 \mu \mathrm{g}$ of protein from each sample was subjected to SDSpolyacrylamide gel electrophoresis (10\% polyacrylamide) and was transferred to a polyvinylidene fluoride membrane. The polyvinylidene fluoride membrane was blotted with a primary antibody as specified in each case. After exposure to the primary antibodies, blots were incubated with the corresponding horseradish peroxidase-conjugated secondary antibodies (Santa Cruz Biotechnology). The reactive bands were visualized with enhanced chemiluminescence substrates and analyzed using Image (National Institutes of Health, Bethesda, MD, USA).

\section{Immunoprecipitation}

Cells were stimulated with Ang II (100 nM), Ang II plus $10 \mathrm{~nm}$ or $100 \mathrm{~nm}$ apelin at $37^{\circ} \mathrm{C}$ in serum-free media for $2 \mathrm{~min}$. The whole-cell lysate from the RIPA buffer was incubated with anti-APJ antibody or anti-rabbit immunoglobulin G antibody overnight at $4{ }^{\circ} \mathrm{C}$ for immunoprecipitation and incubated with protein G agarose (GE Healthcare, Piscataway, NJ, USA) for an additional $3 \mathrm{~h}$ at $4{ }^{\circ} \mathrm{C}$, followed by a brief centrifugation on a bench-top centrifuge. The pellet was washed three times in RIPA buffer and dissolved in the SDSpolyacrylamide gel electrophoresis-loading buffer. Immunoblotting of the heterodimer was performed using an anti- $\mathrm{AT}_{1}$ antibody.

\section{Statistical analysis}

All data were expressed as a mean \pm s.e.m. The data were analyzed using a Student's $t$-test for parametric data or a two-way analysis of variance with Tukey-Kramer multiple comparison tests for comparing multiple groups. A value of $P<0.05$ was considered statistically significant.

\section{RESULTS}

\section{Relationship between apelin-APJ and Ang II-AT ${ }_{1}$ signaling}

To investigate the relationship between apelin-APJ and Ang II-AT signaling, ERK1/2 phosphorylation was evaluated in HEK293 cells transfected with $\mathrm{AT}_{1}$ alone or dual transfected with $\mathrm{AT}_{1}$ and $\mathrm{APJ}$. There was no significant difference in the expression level of $\mathrm{AT}_{1}$ in either of the transfected cell lines. In HEK293 cells dual transfected with $\mathrm{AT}_{1}$ and $\mathrm{APJ}$, ERK1/2 phosphorylation was significantly reduced compared with that of $\mathrm{AT}_{1}$ alone on Ang II stimulation $(P<0.05$ at 2, 5 and $10 \mathrm{~min}$; Figure 1a). However, apelin stimulation dramatically increased the ERK1/2 phosphorylation level beyond the level of $\mathrm{AT}_{1}$ alone $(P<0.05$ at 2 and $5 \mathrm{~min}$; Figure 1a). To further support the idea that non-activated APJ suppresses $\mathrm{AT}_{1}$, PTX was used to create the pharmacologically non-activated APJ. Interestingly, pretreatment with PTX attenuated the ERK1/2 phosphorylation level by apelin, resulting in the same level of ERK1/2 phosphorylation in $\mathrm{AT}_{1}$ and APJ that was observed without the ligand (Figure 1a). Although immunoprecipitation of these receptors revealed that $\mathrm{AT}_{1}$ and $\mathrm{APJ}$ formed heterodimers, apelin stimulation never commuted their heterodimerization (Figure 1b).

\section{Specificity of PTX}

To determine the relationship between apelin-APJ and Ang II-AT ${ }_{1}$, several experiments were performed. Apelin evoked ERK1/2 phosphorylation via an APJ peak at $2 \mathrm{~min}$ and returned to the control level at $10 \mathrm{~min}$ (Figure 2a). In Figure 1a, the peak level of ERK1/2 phosphorylation of Ang II-AT 1 plus apelin-APJ was beyond that of Ang II-AT 1 because of the level of ERK1/2 phosphorylation of apelinAPJ. $\mathrm{AT}_{1}$ coexpression did not affect the ERK1/2 phosphorylation induced by apelin-APJ (Figure 2a). The ERK1/2 and Akt phosphorylation via APJ on apelin stimulation were completely inhibited by pretreatment with PTX, whereas the ERK1/2 phosphorylation through Ang II-AT ${ }_{1}$ was not attenuated by PTX (Figures $2 \mathrm{~b}-\mathrm{d}$ ).

\section{Crosstalk between $\mathrm{AT}_{1}$ and other GPCRs}

To confirm the specificity of APJ for Ang II-AT ${ }_{1}$ signaling, the combination of $\mathrm{AT}_{1}$ and other GPCRs was evaluated. HEK293 cells dual transfected with $\mathrm{AT}_{1}$ and $\beta_{2} \mathrm{AR}$ were generated with the same $\mathrm{AT}_{1}$ expression level. The ERK1/2 phosphorylation level of the dualtransfected HEK293 cells was not significantly different from that of 


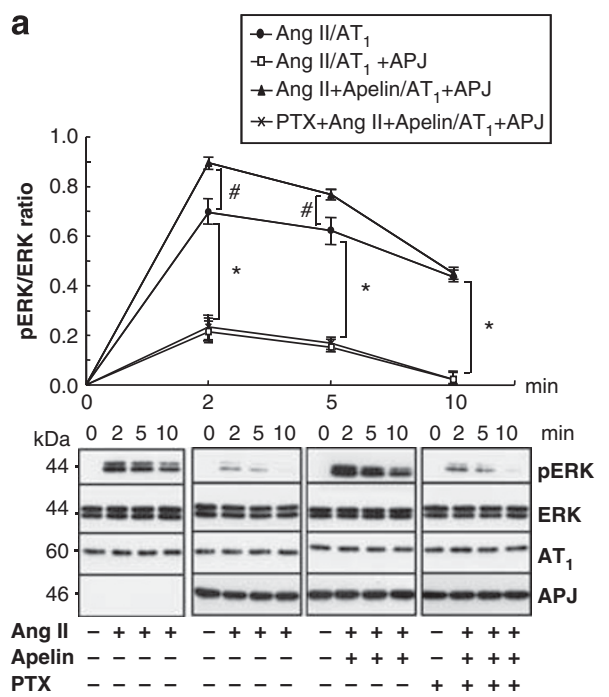

b

WB: anti-pERK

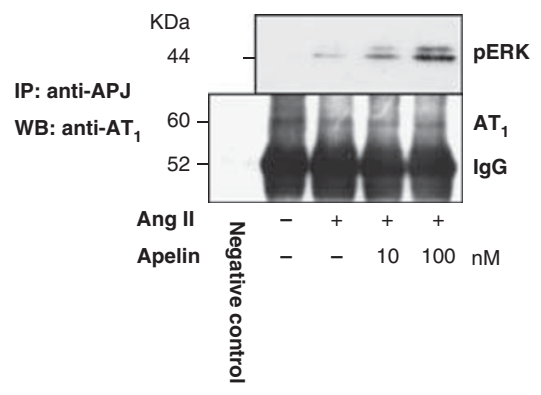

Figure 1 The relationship between apelin-APJ and AT 1 signaling. (a) HEK293 cells that expressed $\mathrm{AT}_{1}$ alone or $A T_{1}$ plus APJ with or without PTX pretreatment were stimulated by Ang II or Ang II plus apelin as indicated. pERK1/2, ERK1/2, AT 1 and APJ were detected by western blot. (b) HEK293 cells that expressed $\mathrm{AT}_{1}$ plus APJ were stimulated by Ang II or Ang II plus apelin as indicated. pERK1/2 was detected by western blot. Immunoprecipitation was performed using an anti-APJ antibody, followed by western blot using an anti-AT ${ }_{1}$ antibody. Data represent the mean \pm s.e.m. from three independent experiments $\left({ }^{*} A n g\right.$ II-AT $1+$ APJ, "Ang II+apelin-AT $1+A P J, P<0.05$ vs. Ang II-AT ${ }_{1}$ ). The molecular weights are indicated in kilodaltons $(\mathrm{kDa})$.

a

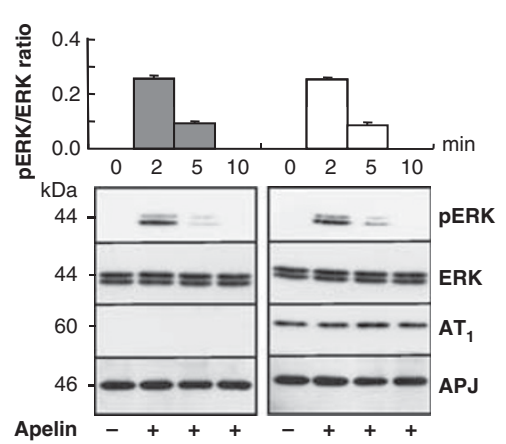

C

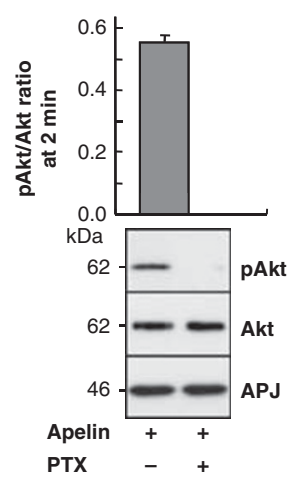

\section{b}
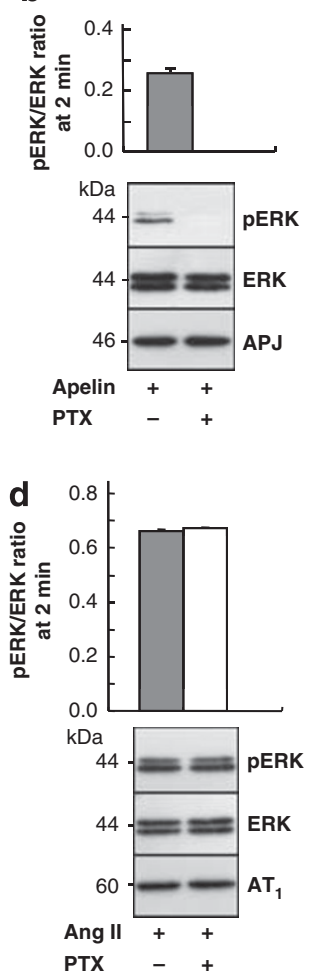

Figure 2 Specificity of PTX. (a) HEK293 cells that expressed APJ alone or $\mathrm{AT}_{1}$ plus APJ were stimulated by apelin as indicated. (b, c) HEK293 cells that expressed APJ upon apelin stimulation for $2 \mathrm{~min}$ in the absence or presence of PTX pretreatment. (d) HEK293 cells that expressed $\mathrm{AT}_{1}$ upon Ang II stimulation for 2 min with or without PTX pretreatment. pERK1/2, ERK1/2, pAkt, Akt, $\mathrm{AT}_{1}$ and APJ were detected by western blot. Data represent the mean \pm s.e.m. from three independent experiments.
$\mathrm{AT}_{1}$ alone on Ang II stimulation (Figure 3a). Next, we evaluated the relationship between $\mathrm{AT}_{1}$ and $\mathrm{AT}_{2}$. Because $\mathrm{AT}_{1}$ and $\mathrm{AT}_{2}$ share the same ligand, the $\mathrm{AT}_{2}$-specific antagonist, $\mathrm{PD} 123319$, was utilized to make the pharmacologically non-activated $\mathrm{AT}_{2}$. The coexpression of non-activated $\mathrm{AT}_{2}$ impacted the ERK1/2 phosphorylation level by the action of Ang II-AT 1 (Figure $3 \mathrm{~b}$ ).

\section{Relationship among apelin, APJ and ARB}

To reveal whether APJ may affect $\mathrm{AT}_{1}$ blocking by ARB, TA-606A was used, and the ERK1/2 phosphorylation level at 2 min after Ang II stimulation was evaluated at various concentrations of TA-606A pretreatment (Figure 4). In HEK293 cells transfected with $\mathrm{AT}_{1}$ alone, pretreatment with $30 \mathrm{~nm}$ of TA-606A significantly decreased the ERK1/2 phosphorylation level $(50 \%$ decrease, $P<0.05)$. Meanwhile, in the HEK293 cells dual transfected with $\mathrm{AT}_{1}$ and non-activated APJ without ligand or PTX, the ERK1/2 phosphorylation level was decreased by $60-61 \%$ of that of $\mathrm{AT}_{1}$ alone without TA-606A, and pretreatment with $30 \mathrm{~nm}$ TA-606A decreased the ERK1/2 phosphorylation level by $73-75 \%$. In contrast, in the cells dual transfected with $\mathrm{AT}_{1}$ and apelin-activated $\mathrm{APJ}$, pretreatment with 120 nм TA-606A only suppressed the ERK1/2 phosphorylation level by $34 \%$ compared with $\mathrm{AT}_{1}$ alone without TA-606A (Figure 4).

\section{DISCUSSION}

The present study demonstrated that non-activated APJ specifically suppressed Ang II-AT 1 signaling. Apelin stimulation eliminated this ligand-independent function of APJ, and the Gi/o-specific inhibitor PTX elicited the pharmacologically non-activated APJ upon apelin stimulation out of relation to its heterodimerization.

\section{Relationship between $\mathrm{AT}_{1}$ and $\mathrm{APJ}$}

Non-activated APJ significantly suppressed Ang II-AT $_{1}$ function, whereas apelin-activated APJ attenuated this suppression. Although Chun et al. never mentioned the relevance between the non-activating and the activating $\mathrm{APJ}$, they demonstrated that $\mathrm{AT}_{1}$ and APJ form receptor heterodimers in the transfected HEK293 

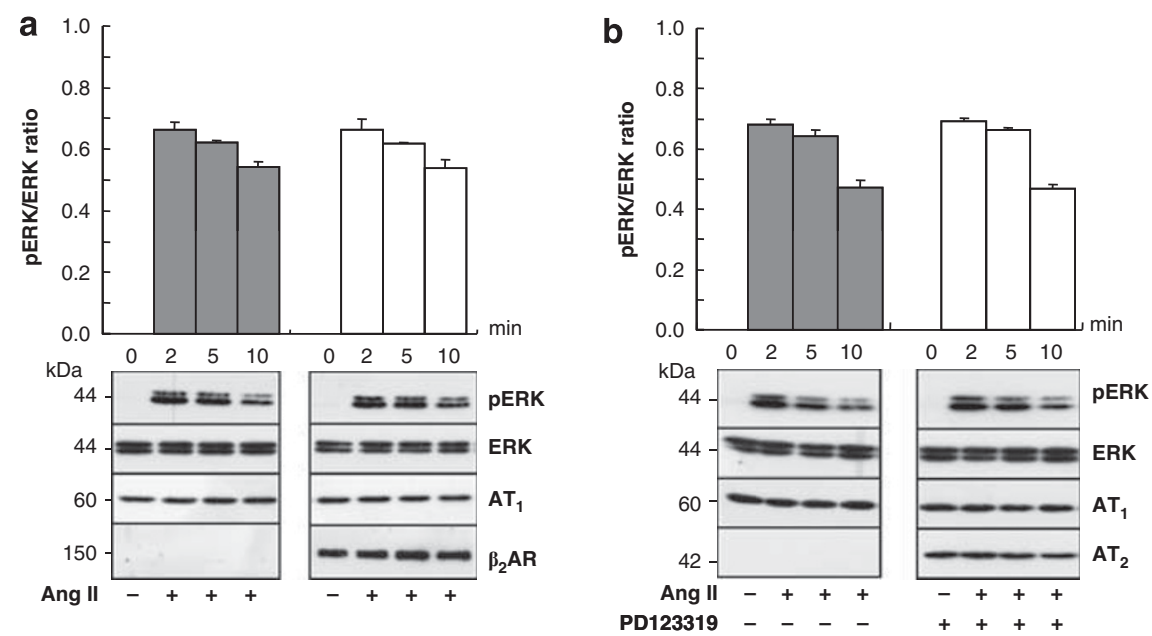

Figure 3 Crosstalk between $A T_{1}$ and other GPCRs. (a) HEK293 cells that expressed $A T_{1}$ alone or $A T_{1}$ plus $\beta_{2} A R$ were stimulated by Ang II as indicated. (b) $\mathrm{AT}_{1}$-expressing HEK293 cells and PD123319-pretreated $\mathrm{AT}_{1}$ - and $\mathrm{AT}_{2}$-coexpressing HEK293 cells were stimulated by Ang II as indicated. pERK1/2, ERK1/2, $A T_{1}, A T_{2}$ and $\beta_{2} A R$ were probed by western blot. Data represent the mean \pm s.e.m. from three independent experiments.

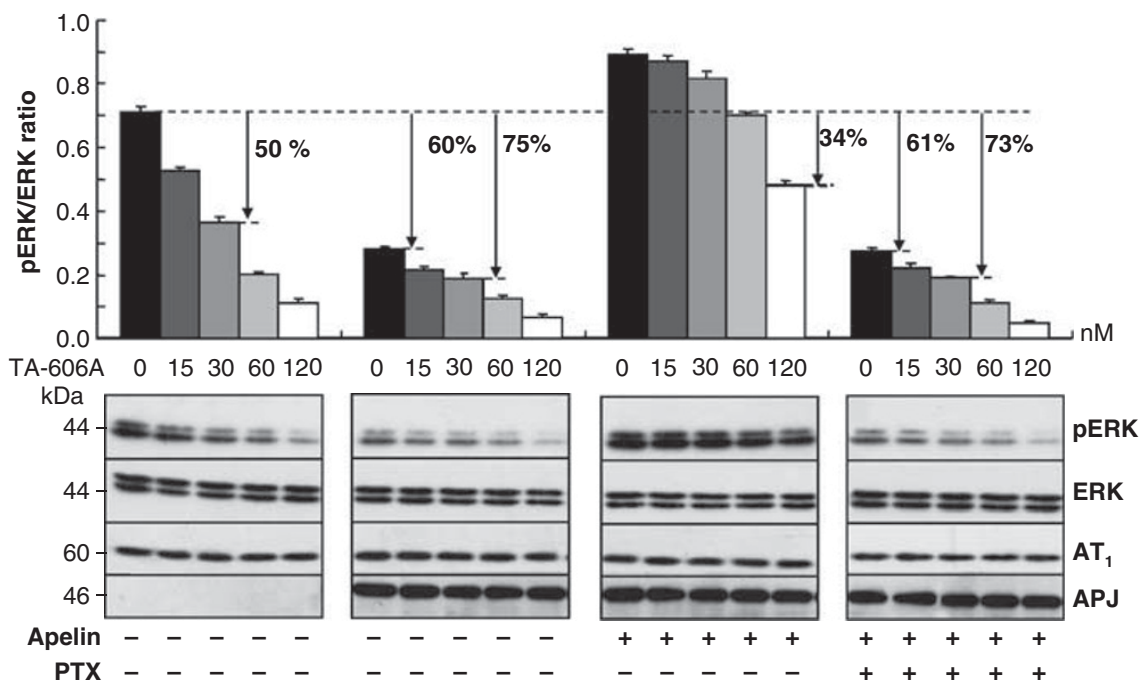

Figure 4 The relationship among apelin, APJ and ARB. HEK293 cells that expressed $\mathrm{AT}_{1}$ alone or AT 1 plus APJ were pretreated with a designed concentration of TA-606A with or without a combination of PTX pretreatment as indicated. Next, cells were simulated by Ang II or Ang II plus apelin for 2 min. pERK1/2, ERK1/2, AT 1 and APJ were detected by western blot. Data represent the mean \pm s.e.m. from three independent experiments (the decreased percentage of the ERK1/2 phosphorylation level with Ang II-AT 1 without TA-606A is indicated).

cells. ${ }^{12}$ Most GPCRs, traditionally considered to function as monomers, actually create homodimers and heterodimers with other GPCRs. These GPCRs' oligomerization may exist in native tissues and may have important consequences to receptor functions. ${ }^{19}$ Interestingly, apelin stimulation never altered the heterodimerization of $\mathrm{APJ}$ and $\mathrm{AT}_{1}$, despite changing their function; however, we also demonstrated that $\mathrm{APJ}$ and $\mathrm{AT}_{1}$ formed receptor heterodimers in the transfected HEK293 cells by immunoprecipitation. Accumulating evidences of the heterodimerization of GPCRs show that heterodimerization may change their receptor functions. Levoye et al. reported that the orphan GPCR, GPR50 receptor, specifically inhibits melatonin receptor type $1 \mathrm{~A}$ function through heterodimerization independently of its ligand. ${ }^{20}$ Occasionally agonists stabilize and/or increase the level of dimers, but they can have the opposite effect. ${ }^{21,22}$ Gines et al. reported that dopamine $\mathrm{D}_{1}$ and adenosine $\mathrm{A}_{1}$ receptors constitutively form heterodimers in the Ltk-fibroblast cells, and the dopamine $\mathrm{D}_{1}$ receptor agonist causes a decrease in the heterodimer level, leading to a cyclic adenosine monophosphate increase. ${ }^{23}$ However, we need to distinguish the relationship between $\mathrm{AT}_{1}$ and APJ from the heterodimerization of other GPCRs. Apelin stimulation may change $\mathrm{AT}_{1}$ function through $\mathrm{APJ}$ without its conversion to heterodimers. Furthermore, the Gi/o inhibitor PTX was used to make a functional non-activated APJ. ${ }^{24-26}$ PTX-induced, pharmacologically non-activated APJ could also suppress the Ang II-AT ${ }_{1}$ signaling, as was the case with the physiologically non-activated APJ. Although the detailed mechanisms of this relationship have been studied, this study 


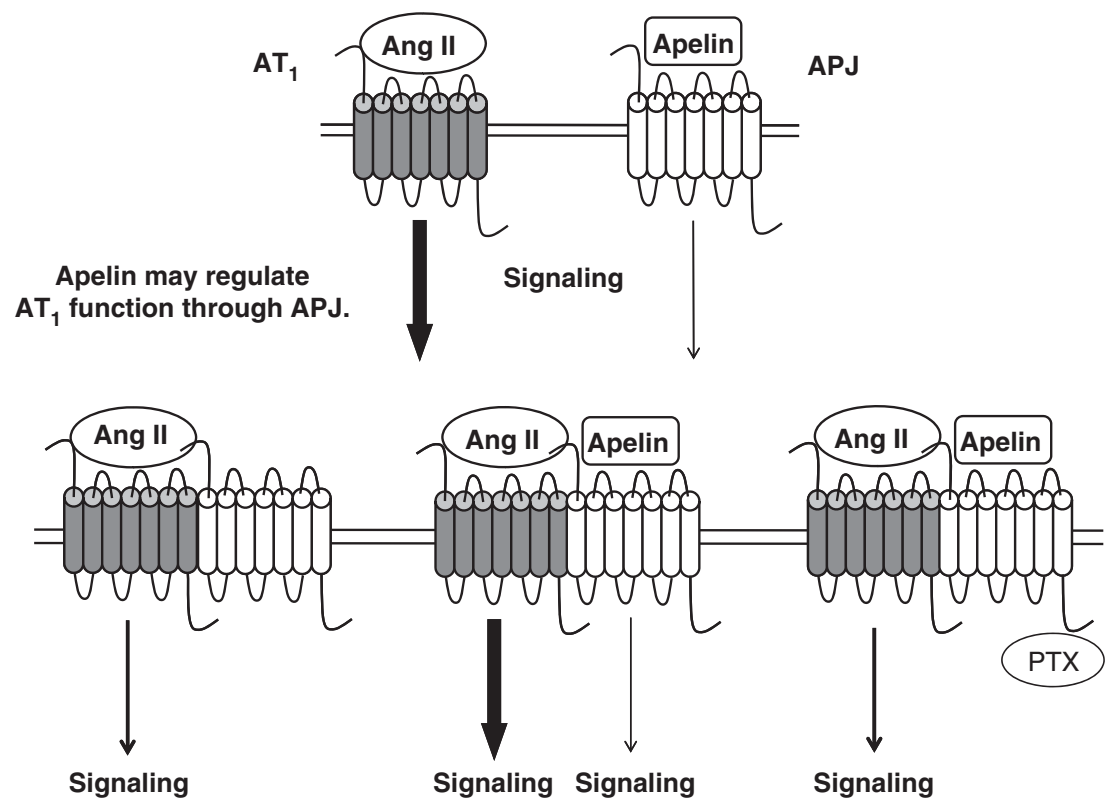

Figure 5 The relationship between APJ and $\mathrm{AT}_{1}$. Both receptors form the heterodimers. Non-activated APJ suppresses $\mathrm{AT}_{1}$ signaling, whereas apelin diminishes this suppression. PTX reverses apelin function.

is the first to demonstrate that the crosstalk between APJ and $\mathrm{AT}_{1}$ was determined by whether or not they received apelin stimulation independently of their heterodimerization. These findings possibly suggest that non-activated APJ, rather than apelin-APJ signaling, had a critical role in inhibiting Ang II-AT ${ }_{1}$. Various accumulating evidences clearly imply that apelin-APJ signaling has the dual functions of cardioprotection and pathologic progression. This study's results suggested that even if the apelin-APJ signaling elicits NO production via activation of endothelial NO synthase leading to cardioprotection, apelin-activated APJ loses Ang II-AT 1 suppression. Taken together, these evidences indicated that apelin-APJ may have dual functions through the regulation of Ang II-AT 1 (Figure 5). When NO production via apelin-APJ signaling is not enough to neutralize the nonactivating APJ function that suppresses Ang II-AT 1 , apelin-APJ signaling may demonstrate its pathological progressive effects. Alternatively, it may behave positively, as is the case with its protective character when there is sufficient NO production.

$\mathrm{AT}_{1}$ was shown to have interactions with several GPCRs in a functional manner. ${ }^{27-30}$ Barki-Harrington et al. reported that interactions exist between $\mathrm{AT}_{1}$ and $\beta_{2} \mathrm{AR}$, which are not affected by ligand stimulation, and a single receptor antagonist effectively blocks downstream signaling and trafficking of both receptors simultaneously. ${ }^{28}$ In our study, the coexpression of $\beta_{2} \mathrm{AR}$ did not affect Ang II-AT signaling. $\mathrm{AT}_{2}$ works as an $\mathrm{AT}_{1}$ antagonist. ${ }^{29,30}$ However, the pharmacologically non-activated $\mathrm{AT}_{2}$ did not affect the Ang II-AT 1 signaling either. Miura et al. also demonstrated that the $\mathrm{AT}_{1}$ signaling may be mainly blocked by the $\mathrm{AT}_{2}$ signaling through their negative crosstalk in phospholipase- $\beta_{3}$ phosphorylation rather than by heterodimerization of both receptors on the cell surface. ${ }^{31}$ Collectively, the ligandindependent function of $\mathrm{APJ}$ in suppressing $\mathrm{AT}_{1}$ signaling may be specific.

\section{Role of apelin-APJ in inhibiting Ang II-AT 1 by ARB}

ARBs, the effective $\mathrm{AT}_{1}$ uncompetitive antagonists, have been proven to be safe and well tolerated for chronic use and work as a critical component of therapy for hypertension, atherosclerosis and heart diseases. ${ }^{32-34}$ ARBs also prolong life in humans. ${ }^{35,36}$ In the present study, the relationship among apelin, APJ and Ang II-AT 1 suggested that the non-activated APJ may work as an endogenous $\mathrm{AT}_{1}$ antagonist, whereas apelin stimulation attenuated this suppression. Therefore, endogenously enhanced ARB efficiency through the non-activated APJ became a substantial pathway for consideration. The enhancing effect of the non-activated APJ on ARBs immediately converted to an abating effect by apelin stimulation. Consequently, our findings may signify that APJ alone but not apelin-APJ signaling may contribute to inhibit Ang $\mathrm{II}_{-} \mathrm{AT}_{1}$ by ARB. Taken together, considering the antagonizing Ang II- $\mathrm{AT}_{1}$ effect of $\mathrm{APJ}$ with/without apelin, the expression level of APJ and apelin in the damaged cardiovascular cells, such as the endothelial cells and VSMC, may have a potential contribution to ARB treatment in the clinical setting.

In summary, the present study has demonstrated that APJ specifically suppresses Ang II-AT 1 signaling, whereas apelin stimulation inhibits this ligand-independent function. These results suggest that the crosstalk between $\mathrm{APJ}$ and $\mathrm{AT}_{1}$ may be distinguishable with or without apelin stimulation. Moreover, we showed that the similarities between apelin-APJ and Ang II-AT ${ }_{1}$ were also reflected in ARB function, and may contribute to ARB treatment in the clinical setting.

\section{CONFLICT OF INTEREST}

The authors declare no conflict of interest.

\section{ACKNOWLEDGEMENTS}

We thank Dr Makoto Endo for valuable suggestions and critical reading of the paper. This study was supported in part by the Takeda Science Foundation, the Maruki Memorial Foundation, research grant HL58205 from the NIH (to TI) and the Saitama Medical University grant (to TS).

1 O'Dowd BF, Heiber M, Chan A, Heng HH, Tsui LC, Kennedy JL, Shi X, Petronis A, George SR, Nguyen T. A human gene that shows identity with the gene encoding the angiotensin receptor is located on chromosome 11. Gene 1993; 136: 355-360. 
2 Tatemoto K, Hosoya M, Habata Y, Fujii R, Kakegawa T, Zou MX, Kawamata Y, Fukusumi S, Hinuma S, Kitada C, Kurokawa T, Onda H, Fujino M. Isolation and characterization of a novel endogenous peptide ligand for the human APJ receptor. Biochem Biophys Res Commun 1998; 251: 471-476.

3 Hosoya M, Kawamata Y, Fukusumi S, Fujii R, Habata Y, Hinuma S, Kitada C, Honda S, Kurokawa T, Onda H, Nishimura O, Fujino M. Molecular and functional characteristics of APJ. Tissue distribution of mRNA and interaction with the endogenous ligand apelin. J Biol Chem 2000; 275: 21061-21067.

4 Kleinz MJ, Skepper JN, Davenport AP. Immunocytochemical localisation of the apelin receptor APJ, to human cardiomyocytes, vascular smooth muscle and endothelial cells. Regul Pept 2005; 126: 233-240.

5 Kleinz MJ, Davenport AP. Emerging roles of apelin in biology and medicine. Pharmacol Ther 2005; 107: 198-211.

6 Lee DK, George SR, O'Dowd BF. Unravelling the roles of the apelin system: prospective therapeutic applications in heart failure and obesity. Trends Pharmacol Sci 2006; 27: 190-194.

7 Kidoya $\mathrm{H}$, Naito $\mathrm{H}$, Takakura N. Apelin induces enlarged and non-leaky blood vessels for functional recovery from ischemia. Blood 2010; 115: 3166-3174.

8 Farkasfalvi K, Stagg MA, Coppen SR, Siedlecka U, Lee J, Soppa GK, Marczin N, Szokodi I, Yacoub MH, Terracciano CM. Direct effects of apelin on cardiomyocyte contractility and electrophysiology. Biochem Biophys Res Commun 2007; 357: 889-895.

9 Japp AG, Cruden NL, Barnes G, van Gemeren N, Mathews J, Adamson J, Johnston NR, Denvir MA, Megson IL, Flapan AD, Newestern bloty DE. Acute cardiovascular effects of apelin in humans: potential role in patients with chronic heart failure. Circulation 2010; 121: $1818-1827$.

10 Iwanaga Y, Kihara Y, Takenaka H, Kita T. Down-regulation of cardiac apelin system in hypertrophied and failing hearts: possible role of angiotensin II-angiotensin type 1 receptor system. J Mol Cell Cardiol 2006; 41: 798-806.

11 Ishida J, Hashimoto T, Hashimoto Y, Nishiwaki S, Iguchi T, Harada S, Sugaya T, Matsuzaki H, Yamamoto R, Shiota N, Okunishi H, Kihara M, Umemura S, Sugiyama F, Yagami K, Kasuya Y, Mochizuki N, Fukamizu A. Regulatory roles for APJ a seven-transmembrane receptor related to angiotensin-type 1 receptor in blood pressure in vivo. J Biol Chem 2004; 279: 26274-26279.

12 Chun HJ, Ali ZA, Kojima Y, Kundu RK, Sheikh AY, Agrawal R, Zheng L, Leeper NJ, Pearl NE, Patterson AJ, Anderson JP, Tsao PS, Lenardo MJ, Ashley EA, Quertermous T. Apelin signaling antagonizes Ang II effects in mouse models of atherosclerosis. J Clin Invest 2008; 118: 3343-3354.

13 Hashimoto T, Kihara M, Imai N, Yoshida S, Shimoyamada H, Yasuzaki H, Ishida J, Toya Y, Kiuchi Y, Hirawa N, Tamura K, Yazawa T, Kitamura H, Fukamizu A, Umemura S. Requirement of apelin-apelin receptor system for oxidative stress-linked atherosclerosis. Am J Pathol 2007; 171: 1705-1712.

14 Liu C, Su T, Li F, Li L, Qin X, Pan W, Feng F, Chen F, Liao D, Chen L. PI3K/Akt signaling transduction pathway is involved in rat vascular smooth muscle cell proliferation induced by apelin-13. Acta Biochim Biophys Sin 2010; 42: 396-402.

15 Yoshida T, Mishina M. Neuron-specific gene manipulations to transparent zebrafish embryos. Methods Cell Sci 2003; 25: 15-23.

16 Kawai M, Maruyama H, Bessho K, Yamamoto H, Miyazaki J, Yamamoto T. Simple strategy for bone regeneration with a $B M P-2 / 7$ gene expression cassette vector. Biochem Biophys Res Commun 2009; 390: 1012-1027.

17 Li Z, Zhang H, Lundin L, Thullberg M, Liu Y, Wang Y, Claesson-Welsh L, Stromblad S. p21-activated kinase 4 phosphorylation of integrin beta5 Ser-759 and Ser-762 regulates cell migration. J Biol Chem 2010; 285: 23699-23710.
18 Hashimoto Y, Harada Y, Narita H, Naito K, Murata S. The pharmacologic profile of 606A, a novel angiotensin II receptor antagonist. J Cardiovasc Pharmacol 1997; 29: 284-290.

19 Prinster SC, Hague C, Hall RA. Heterodimerization of g protein-coupled receptors: specificity and functional significance. Pharmacol Rev 2005; 57: 289-298.

20 Levoye A, Dam J, Ayoub MA, Guillaume JL, Couturier C, Delagrange P, Jockers R. The orphan GPR50 receptor specifically inhibits MT1 melatonin receptor function through heterodimerization. EMBO J 2006; 25: 3012-3023.

21 Terrillon S, Bouvier M. Roles of G-protein-coupled receptor dimerization. EMBO Rep 2004; 5: 30-34.

22 Rios CD, Jordan BA, Gomes I, Devi LA. G-protein-coupled receptor dimerization: modulation of receptor function. Pharmacol Ther 2001; 92: 71-87.

23 Gines S, Hillion J, Torvinen M, Le Crom S, Casado V, Canela El, Rondin S, Lew JY, Watson S, Zoli M, Agnati LF, Verniera P, Lluis C, Ferre S, Fuxe K, Franco R. Dopamine $\mathrm{D} 1$ and adenosine $\mathrm{A} 1$ receptors form functionally interacting heteromeric complexes. Proc Natl Acad Sci USA 2000; 97: 8606-8611.

24 Masri B, Lahlou H, Mazarguil H, Knibiehler B, Audigier Y. Apelin (65-77) activates extracellular signal-regulated kinases via a PTX-sensitive G protein. Biochem Biophys Res Commun 2002; 290: 539-545.

25 Masri B, Morin N, Pedebernade L, Knibiehler B, Audigier Y. The apelin receptor is coupled to Gi1 or Gi2 protein and is differentially desensitized by apelin fragments. J Biol Chem 2006; 281: 18317-18326.

26 Hamada J, Kimura J, Ishida J, Kohda T, Morishita S, Ichihara S, Fukamizu A. Evaluation of novel cyclic analogues of apelin. Int J Mol Med 2008; 22: 547-552.

27 AbdAlla S, Lother H, el Massiery A, Quitterer U. Increased AT(1) receptor heterodimers in preeclampsia mediate enhanced angiotensin II responsiveness. Nat Med 2001; 7: 1003-1009.

28 Barki-Harrington L, Luttrell LM, Rockman HA. Dual inhibition of beta-adrenergic and angiotensin II receptors by a single antagonist: a functional role for receptor-receptor interaction in vivo. Circulation 2003; 108: 1611-1618.

29 AbdAlla S, Lother H, Abdel-tawab AM, Quitterer U. The angiotensin II AT2 receptor is an AT1 receptor antagonist. J Biol Chem 2001; 276: 39721-39726.

30 Horiuchi M, Hayashida W, Akishita M, Tamura K, Daviet L, Lehtonen JY, Dzau VJ. Stimulation of different subtypes of angiotensin II receptors AT1 and AT2 receptors, regulates STAT activation by negative crosstalk. Circ Res 1999; 84: 876-882.

31 Miura S, Matsuo Y, Kiya Y, Karnik SS, Saku K. Molecular mechanisms of the antagonistic action between AT1 and AT2 receptors. Biochem Biophys Res Commun 2010; 391: 85-90.

32 Eberhardt RT, Kevak RM, Kang PM, Frishman WH. Angiotensin II receptor blockade: an innovative approach to cardiovascular pharmacotherapy. J Clin Pharmacol 1993; 33: 1023-1038.

33 Israili ZH. Clinical pharmacokinetics of angiotensin II (AT1) receptor blockers in hypertension. J Hum Hypertens 2000; 14 (Suppl 1): S73-S86.

34 Wassmann S, Czech T, van Eickels M, Fleming I, Böhm M, Nickenig G. Inhibition of diet-induced atherosclerosis and endothelial dysfunction in apolipoprotein E/angiotensin II type 1A receptor double-knockout mice. Circulation 2004; 110: 3062-3067.

35 Benigni A, Corna D, Zoja C, Sonzogni A, Latini R, Salio M, Conti S, Rottoli D, Longaretti L, Cassis P, Morigi M, Coffman TM, Remuzzi G. Disruption of the Ang II type 1 receptor promotes longevity in mice. J Clin Invest 2009; 119: 524-530.

36 Cassis P, Conti S, Remuzzi G, Benigni A. Angiotensin receptors as determinants of life span. Pflugers Arch 2010; 459: 325-332. 